\title{
Physical function measures and health-related quality of life in primary care medicine: cross-sectional study
}

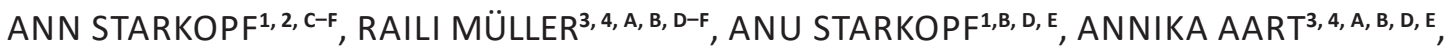

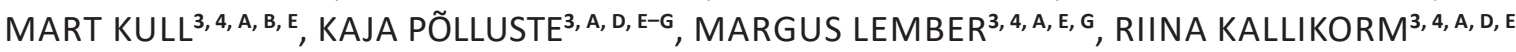

${ }^{1}$ OÜ Perearst Anu Starkopf, Primary Health Care Centre, Tartu, Estonia

${ }^{2}$ Institute of Family Medicine and Public Health, University of Tartu, Estonia

${ }^{3}$ Department of Internal Medicine, University of Tartu, Estonia

${ }^{4}$ Internal Medicine Clinic, Tartu University Hospital, Estonia

A - Study Design, B - Data Collection, C - Statistical Analysis, D - Data Interpretation, E - Manuscript Preparation, F - Literature Search, G - Funds Collection

Summary Background. Health-related quality of life (HRQoL) is an important component of comprehensive management in primary care.

Objectives. The purpose of this study was to investigate the relationships between physical performance measures and self-reported $\mathrm{HRQOL}$ and to find optimal values of muscle function tests associated with lower HRQoL.

Material and methods. From a single primary health care center patient list, 330 subjects were randomly selected. Information about the patient's age, gender, body mass index and presence of self-reported chronic diseases was collected, as well as analyses for systemic inflammation and vitamin D. Physical performance was measured by dominant hand grip strength (GS) and a 30-second chair stand test (30-CST). The physical (PCS) and mental (MCS) component scores of the Short-Form-36 Questionnaire (SF-36) were used to evaluate $\mathrm{HRQ}$ oL. The predictive power of physical function measures were tested with multivariate linear regression analyses. Threshold values for physical function tests were calculated by receiver operating characteristic curves.

Results. Multivariate analyses demonstrated that 30-CST was significantly $(p<0.0001)$ associated with SF-36 summary scores for both genders. Males with 30-CST results under 7 stands and females with results of fewer than 13 stands were in the risk group for having the lowest PCS scores. Results in 30-CST under 12 stands in males and under 13 stands in females were associated with the lowest scores of MCS.

Conclusions. 30-CST had the most expressed association with the outcomes of HRQoL in the Estonian population. Subjects with physical performance results under threshold values are at risk of lower HRQoL; therefore, 30-CST is a potential screening indicator for HRQoL assessment.

Key words: physical function, population, primary care, physical quality of life, mental quality of life, SF-36.

Starkopf A, Müller R, Starkopf A, Aart A, Kull M, Põlluste K, Lember M, Kallikorm R. Physical function measures and health-related quality of life in primary care medicine: cross-sectional study. Fam Med Prim Care Rev 2017; 19(2): 161-166, doi: https://doi.org/10.5114/ fmpcr.2017.67872.

\section{Background}

Health-related quality of life (HRQoL) is an important construct that describes the impact of health conditions on an individual's overall quality of life. One of the core objectives in the comprehensive management of primary care is to improve or at least attain patients' HRQoL. Information obtained from quality of life can be useful for general practitioners to indicate the directions needed for more efficient disease prevention or treatment of patients with chronic diseases [1, 2]. Therefore, HRQoL has become an important indicator in primary care.

Physical capability is one of the factors that is associated with HRQoL [3]. Moreover, low physical capability may indicate health problems, e.g. systemic inflammation and low serum vitamin D concentrations $[4,5]$. Physical function measures and muscle strength have been shown to predict important clinical outcomes [6] and are associated with physical disability and cognitive decline [7]. Grip strength (GS) and the 30-second chair stand test (30-CST) are measures that require minimal space and equipment and could be easily implemented in a general practitioner's office, where the contact time with patients is often limited.
As a result, interest is increasing in physical function tests and their use as simple screening tools to estimate HRQoL in the general population [2]. With these factors in mind, the present study is the first in Estonia to find out the associations between objectively measured physical performance and self-reported HRQoL, as well as potential cut-off values for physical function tests to distinguish people with lower and higher HRQoL.

\section{Material and methods}

\section{Study design}

A cross-sectional study was conducted in collaboration with the Department of Internal Medicine, University of Tartu, and a single primary health care center in Tartu, Estonia. The data of all patients admitted to the research was collected from 1 September 2014 until 31 March 2015. Participation was voluntary and all patients signed a written informed consent form. The study was approved by the Ethics Review Committee on Human Research of the University of Tartu. 


\section{Subjects}

To form the study group, 350 subjects were randomly selected from the primary health care center patients' list (total number of patients 1,854 ), adjusted for the age and gender breakdown of the Estonian population in 2013. Subjects ranged in age from 20 to 79 years, with a mean age of $47.7 \pm 16.3$ years. Firstly, postal invitations with introductory materials were sent out. A total number of 332 subjects contacted the primary health care center for further instructions. All measurements were conducted at the Department of Internal Medicine, University of Tartu, by independent researchers.

\section{Background characteristics and biochemical markers}

General information was collected regarding age, gender and self-reported chronic conditions. Anthropometric data about height and weight was measured before muscle strength tests. Body mass index (BMI) was calculated as weight in kilograms divided by the square of height in meters. Blood samples were taken for the measurement of high-sensitivity C-reactive protein (hsCRP) and vitamin D. High-sensitivity CRP was used as a marker for systemic inflammation.

\section{Physical function measures}

To measure muscle function, we used dominant hand GS and 30-CST. GS was measured in bars with a calibrated Riester Dynatest dynamometer. Participants were tested according to the American Society of Hand Therapists' recommended standardized positioning [8]. One practice attempt was followed by three consecutive attempts with 30- to 60-second pauses in-between. Statistical analysis included the average value of three test trials. For the 30-CST, the patients were asked to sit on a chair with their hands on the opposite shoulder across their chest. Upon a signal, subjects rose to a full standing position and returned to a fully seated position at their own pace for as many repetitions as they could. One practice attempt of 1-3 repetitions was followed by one 30-second trial. The final score of chair stands was the total number of unassisted full stands during the 30-second timeframe. Both physical function measures have shown to be valid, reliable and have good test-retest reliability $[9,10]$.

\section{Health-related quality of life}

The impact of physical function measures on health-related quality of life was evaluated using the validated Estonian version of the Short-Form-36 (SF-36) questionnaire [11]. All participants self-administered the SF- 36 form, and if requested, the help of a health care professional of the Department of Internal Medicine was provided. The SF-36 has eight domains of health, which can be divided into two categories: a physical component summary (PCS) and mental component summary (MCS). In our study, we used only two summary scores to represent the overall estimate of HRQoL.

\section{Statistical analyses}

All statistical analyses were performed using the $R$ software package version 3.1.2 ( $R$ Foundation for Statistical Computing). Descriptive statistics were used to determine demographic and health-related characteristics. Log-transformed values for variables with a right skewed data distribution were used. These variables included hsCRP and vitamin $D$. The data is presented as the number of appearances (percentage) for categorical variables and as the median (interquartile range) for continuous variables. To compare baseline characteristics between male and female patients, we used a $t$-test for continuous variables and the Wilcoxon Rank Sum test for categorical variables. Pearson correlation coefficient was used to assess the agreement between GS and 30-CST measures.

Univariate linear regression analysis for SF-36 summary scores adjusted to physical function measures was performed.
A separate model for each of the summary scores (PCS and MCS) was fitted for both genders. Both physical function measures and parameters, such as age, hsCRP, vitamin D and presence of self-reported chronic disease, were stepwise entered into multiple linear regression models with summary scores of SF-36 as the dependent variable. The strength of regression models was evaluated with determination coefficient $R^{2}$. The variance inflation factor (VIF) was calculated to describe multicollinearity, while fitting a model into a multiple linear regression analysis. To define subjects with low HRQoL, the lower quartile of PCS and MCS was used, based upon which patients were divided in groups (low PCS and MCS, high PCS and MCS). Receiver operating characteristic (ROC) curves and area under curve (AUC) were used to assess the effectiveness of 30-CST and GS. The threshold values of physical function tests were defined according to the optimal cut-off values calculated by ROC for both genders separately. Statistical analyses accepted $p$-values of $<0.05$ for the level of statistical significance.

\section{Results}

\section{Study cohort}

In total, 332 patients were recruited during the study period. Two patients with missing outcome data were excluded from final analysis $(n=330)$. There were significant differences in age, physical function measures, HRQoL and self-reported chronic diseases between males and females. The background characteristics of the study group are presented in Table 1.

\begin{tabular}{|c|c|c|c|}
\hline \multicolumn{4}{|c|}{ Background characteristics } \\
\hline & $\begin{array}{l}\text { Male } \\
(n=154)\end{array}$ & $\begin{array}{l}\text { Female } \\
(n=176)\end{array}$ & \\
\hline & \multicolumn{2}{|c|}{$n(\%)$ or mean (SD) } & $p^{\mathrm{a}}$ \\
\hline \multicolumn{4}{|c|}{ Demographic characteristics } \\
\hline Age (years) & $45.5(15.7)$ & $49.6(16.7)$ & 0.0201 \\
\hline \multicolumn{4}{|l|}{ Weight status $^{\mathrm{b}}$} \\
\hline $\mathrm{BMI}, \mathrm{kg} / \mathrm{m}^{2}$ & $27.5(4.5)$ & $27.0(6.1)$ & 0.3983 \\
\hline $\mathrm{BMI}<25$ & $52(33.8)$ & $74(42.0)$ & \\
\hline BMI 25-29.9 & $56(36.4)$ & $51(29.0)$ & \\
\hline $\mathrm{BMI} \geq 30$ & $46(29.9)$ & $51(29.0)$ & 0.2371 \\
\hline \multicolumn{4}{|c|}{ Biochemical parameters } \\
\hline $\mathrm{hsCRP}, \mathrm{mg} / \mathrm{L}^{\mathrm{c}}$ & $0.7(3.7)$ & $0.9(5.0)$ & 0.4162 \\
\hline Vitamin $\mathrm{D}, \mathrm{nmol} / \mathrm{L}^{\mathrm{c}}$ & $36.6(1.5)$ & $40.4(1.5)$ & 0.3780 \\
\hline \multicolumn{4}{|c|}{ Physical function measures } \\
\hline $\begin{array}{l}\text { Chair stands, no. of } \\
\text { stands in } 30 \text { seconds }\end{array}$ & $19.9(8.4)$ & $15.4(6.5)$ & $<0.001$ \\
\hline $\begin{array}{l}\text { Dominant hand grip } \\
\text { strength, bars }\end{array}$ & $0.7(0.2)$ & $0.3(0.1)$ & $<0.001$ \\
\hline \multicolumn{4}{|c|}{ Health-related quality of life ${ }^{d}$} \\
\hline Physical & $76.9(17.4)$ & $70.6(23.0)$ & 0.0057 \\
\hline Mental & $71.9(19.1)$ & $67.2(20.9)$ & 0.0350 \\
\hline \multicolumn{2}{|c|}{ Self-reported chronic diseases } & & 0.0193 \\
\hline yes & $72(46.8)$ & $106(60.2)$ & \\
\hline no & $82(53.2)$ & $70(39.8)$ & \\
\hline
\end{tabular}

${ }^{a}$ Significance of the difference between males and females; ${ }^{b} \mathrm{BMI}$ - body mass index; ' ${ }^{\mathrm{C}}$ Log-transformed values; hsCRP - high-sensitivity C-reactive protein; ${ }^{d}$ Component summary scores according to the SF-36 Short Form Questionnaire.

The range of stands in 30-CST varied from 0 to 40 for males, while for females, the range was from 0 to 35 stands. The minimum result for males in GS was 0.1 bars, and the maximum result was 1.1 bars; for females, the minimum GS was 0.0 bars, and the maximum was 0.7 bars. 


\begin{tabular}{|c|c|c|c|c|c|c|}
\hline \multicolumn{7}{|c|}{ Physical component summary ${ }^{\mathrm{a}}$} \\
\hline & \multicolumn{3}{|c|}{ Male $(n=154)\left(\mathrm{adj} . R^{2}=0.33\right)$} & \multicolumn{3}{|c|}{ Female $(n=176)\left(\mathrm{adj} . R^{2}=0.38\right)$} \\
\hline Independent variables & Coeff. & Cl. 95 & $p$ & Coeff. & \begin{tabular}{|l} 
Cl. 95 \\
\end{tabular} & $p$ \\
\hline Age & -0.17 & {$[-0.33 ;-0.02]$} & 0.0299 & -0.05 & {$[-0.20 ; 0.09]$} & 0.4730 \\
\hline $\mathrm{BMI}^{\mathrm{b}}$ & 0.02 & {$[-0.43 ; 0.46]$} & 0.9410 & -0.31 & {$[-0.73 ; 0.11]$} & 0.1432 \\
\hline Vitamin $D^{c}$ & -0.20 & {$[-4.86 ; 4.46]$} & 0.9322 & -1.63 & {$[-5.92 ; 2.66]$} & 0.4565 \\
\hline hsCRPc & -2.31 & {$[-3.67 ;-0.94]$} & 0.0010 & -2.21 & {$[-3.61 ;-0.80]$} & 0.0023 \\
\hline Chair stands & 0.88 & {$[0.56 ; 1.21]$} & $<0.0001$ & 0.89 & {$[0.58 ; 1.21]$} & $<0.0001$ \\
\hline Grip strength $^{d}$ & 6.25 & {$[-2.92 ; 15.41]$} & 0.1824 & 17.57 & {$[8.82 ; 26.32]$} & $<0.0001$ \\
\hline Chronic disease $^{\mathrm{e}}$ & -7.42 & {$[-12.28 ;-2.56]$} & 0.0029 & -9.00 & {$[-13.55 ;-4.45]$} & 0.0001 \\
\hline \multicolumn{7}{|c|}{ Mental component summary ${ }^{\mathrm{a}}$} \\
\hline & \multicolumn{3}{|c|}{ Male $(n=154)\left(\right.$ adj. $\left.R^{2}=0.14\right)$} & \multicolumn{3}{|c|}{ Female $(n=176)\left(\mathrm{adj} . R^{2}=0.17\right)$} \\
\hline Independent variables & Coeff. & $\mathrm{Cl} .95$ & $p$ & Coeff. & $\mathrm{Cl} .95$ & $p$ \\
\hline Age & 0.22 & {$[0.05 ; 0.39]$} & 0.0103 & 0.24 & {$[0.09 ; 0.40]$} & 0.0026 \\
\hline $\mathrm{BMI}^{\mathrm{b}}$ & 0.16 & {$[-0.32 ; 0.63]$} & 0.5215 & -0.06 & {$[-0.51 ; 0.39]$} & 0.7916 \\
\hline Vitamin $D^{c}$ & -1.93 & {$[-6.94 ; 3.09]$} & 0.4519 & -2.86 & {$[-7.45 ; 1.74]$} & 0.2244 \\
\hline hsCRPc & -1.93 & {$[-3.39 ;-0.46]$} & 0.0106 & -1.56 & {$[-3.07 ;-0.05]$} & 0.0434 \\
\hline Chair stands & 0.76 & {$[0.41 ; 1.11]$} & $<0.0001$ & 0.67 & {$[0.34 ; 1.01]$} & 0.0001 \\
\hline Grip strength $^{d}$ & 6.67 & {$[-3.19 ; 16.53]$} & 0.1859 & 13.56 & {$[4.18 ; 22.94]$} & 0.0049 \\
\hline Chronic disease $^{\mathrm{e}}$ & -7.89 & {$[-13.12 ;-2.66]$} & 0.0033 & -9.56 & {$[-14.44 ;-4.68]$} & 0.0001 \\
\hline
\end{tabular}

${ }^{a}$ Component summary scores according to the SF-36 Short Form Questionnaire; ${ }^{b}$ BMI - body mass index; ${ }^{c}$ Log-transformed values; hsCRP - highsensitivity C-reactive protein; ${ }^{\mathrm{d}}$ Dominant hand grip strength; ${ }^{\mathrm{e}}$ Self-reported chronic disease: 0 - no; 1 - yes.

\section{Health-related quality of life and physical function}

Univariate analyses demonstrated that 30 -CST and dominant hand GS were significantly $(p<0.0001)$ associated with SF-36 summary scores for male and female patients. The univariate model with 30 -CST explained almost $30 \%$ of PCS variance (male-adjusted $R^{2}=0.26$; female-adjusted $R^{2}=0.28$ ), whereas GS only was around $10 \%$ (male-adjusted $R^{2}=0.10$; female-adjusted $R^{2}=0.17$ ). The physical function measures used in the analyses predicted the variance of PCS better than MCS. GS was moderately correlated with 30-CST, both in males and females $(r=0.44-0.45 ; p<0.0001)$.

The associations between SF-36 summary scores and 30-CST remained significant in multiple linear regression analyses $(p<0.0001)$ for male and female patients. In addition, dominant hand GS remained significant only for female patients $(p<$ 0.0001 ). Multiple regression models accounted for $33 \%$ (adjusted $R^{2}=0.33$ ) in males and $37 \%$ (adjusted $R^{2}=0.38$ ) in females for the observed variability of PCS. As expected, a multiple regression model for PCS was stronger compared with the model for MCS (male-adjusted $R^{2}=0.14$; female-adjusted $R^{2}=0.17$ ).
Independent predictors of SF-36 summary scores on multiple regression analyses are presented in Table 2 . All multiple regression models used in the analyses were not affected by multicollinearity (VIF $\leq 1.6$ ).

Of all subjects $(n=330), 55$ were in the "low PCS" group, and 63 were in the "low MCS" group. The lower quartile for PCS was a value of 49 , and for MCS, a value of 48 on a scale from 0 to 100 , whereas higher values in the SF-36 component summary scale are related to higher HRQoL. We found 30-CST to be a better diagnostic test for evaluation of patients who might be at risk of lower HRQoL. According to AUC values, the diagnostic accuracy of 30-CST for PCS was significantly better compared to GS for males $(p=0.015)$ and females $(p=0.001)$. There was no statistically significant difference for males $(p=0.241)$ and females $(p=0.158)$ in the diagnostic accuracy of physical function tests for MCS. ROC curves and AUC values for 30-CST are presented in Figure 1 and 2. The optimal cut-off values of 30-CST calculated by the ROC curve to predict low PCS, defined as the lower quartile of PCS, were 7 chair stands for males and 13 chair stands for females. For low MCS, the optimal cut-off values were 12 chair stands for males and 13 chair stands for females.

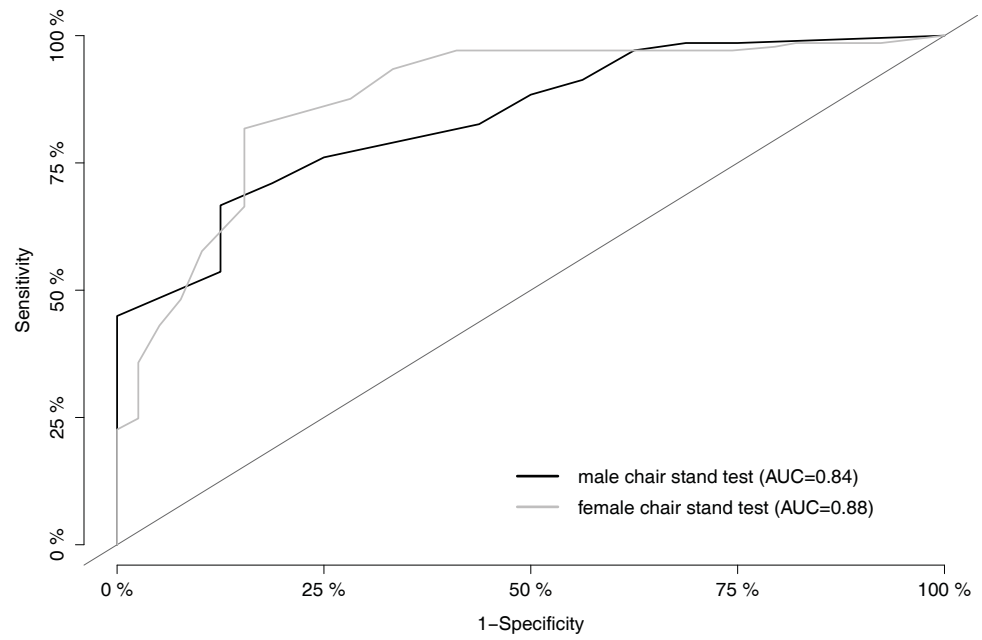

Figure 1. ROC curves for the 30-second chair stand test to predict low physical component summary of health-related quality of life 


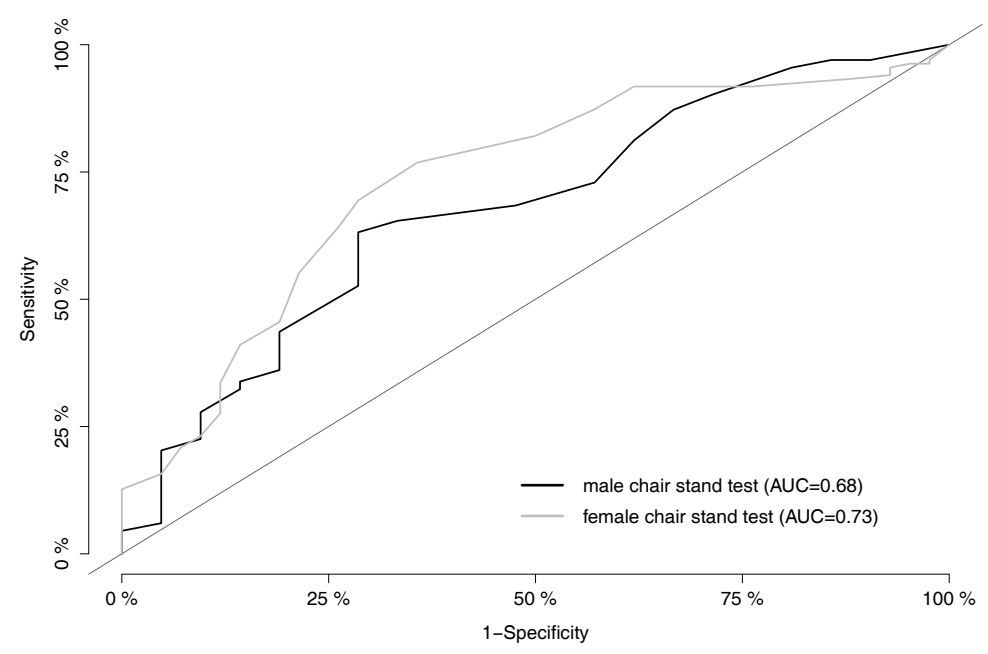

Figure 2. ROC curves for the 30-second chair stand test to predict low mental component summary of health-related quality of life

\section{Self-reported chronic diseases}

$54 \%(n=178)$ of the subjects reported a chronic condition, with unequal gender distribution (72 men and 106 women; $p=$ 0.0193). Several patients reported more than one chronic condition. Self-reported chronic diseases most frequently included hypertension, arrhythmia, osteoarthritis, diabetes, hypercholesterolemia, hypothyroidism, asthma, malignancy and osteoporosis.

The mean age of participants with chronic diseases was $55.8 \pm 15.3$ years, and in the healthy group, $38.2 \pm 11.9$ years. There was no statistically significant $(p=0.5988)$ difference in vitamin $D$ values between the two groups. The majority of the study group - 228 patients $(71 \%)$ - had vitamin D serum levels $\leq 50 \mathrm{nmol} / \mathrm{L}$, indicating insufficiency, and there was no significant difference between gender $(p=0.2215)$. Only $4 \%(n=12)$ of the subjects, 4 men and 8 women, had a vitamin $D$ value over the optimal level of $75 \mathrm{nmol} / \mathrm{L}$.

As expected, both physical function measures were substantially better $(p<0.001)$ in subjects who reported as not having any chronic diseases. The mean number of stands in 30-CST in the healthy group was $21.6 \pm 6.9$, and the mean GS was 0.5 \pm 0.2 bars. In the group with chronic diseases, the mean results were $14.1 \pm 6.8$ stands in 30-CST and $0.4 \pm 0.2$ bars in GS. Based on the reported $p$-value $(<0.0001)$, we can conclude that there was a statistically significant difference in PCS and MCS, with a median difference of 16 points $(\mathrm{Cl} .95 \%=[12 ; 20])$ for $\mathrm{PCS}$ and 8 points $(\mathrm{Cl} .95 \%=[4 ; 13])$ for MCS between subjects with chronic disease and the healthy group.

\section{Discussion}

In this study, the associations between physical function tests and self-reported $\mathrm{HRQ}$ oL depended on the muscle strength measure used. The key finding is that 30-CST was most strongly associated with HRQoL, slightly more with physical than mental health status. The observed positive associations between 30-CST and PCS/MCS seem to be independent of BMI and vitamin $D$ for both genders, while age, chronic conditions and higher markers of systemic inflammation remained dependent. The possible reason why the association between vitamin $D$ and physical capability or HRQoL was not found might be the equally low level of vitamin $\mathrm{D}$ among the Estonian population [12]. We found 30-CST to be a better diagnostic test for evaluating patients who might be at risk for lower HRQoL. In the Estonian population, male results under 7 stands and female results under 13 stands were associated with the lowest scores of PCS, while results in 30-CST under 12 stands in males and 13 stands in females were associated with the lowest scores of MCS.

In support of the previous results [3], a positive association between muscle strength and self-reported HRQoL was demonstrated in our study. Increased functional performance permits people's autonomy for a more dynamic and socially active lifestyle and therefore increased quality of life. There is also evidence that better lower-extremity performance is associated with reduced risk of mortality and other adverse health outcomes [6]. Cooper et al. have suggested that better physical capability is associated with subsequent positive mental well-being. This association is explained with an increased risk of lower physical function to disability, which has been shown to have a negative influence on mental well-being [13]. Still, it cannot be ruled out that mental and physical health quality across age groups could be influenced by other factors (socioeconomic status, social support, absence of severe diseases, cognitive capability).

Hall et al. [14] have reported that male upper body physical function is substantially more related to mental than physical HRQoL, while for lower extremity function, it applies conversely. In contrast, our current study revealed no associations between GS and self-reported physical and mental HRQoL in adjusted models (adjustment for age, BMI, hsCRP, vitamin D and chronic conditions) among men. Although our data does not allow for a more detail examination, we assume that the discrepancy between physical function tests and HRQoL is explained with the stronger impact of lower muscle strength on daily activities. Most of our study group was comprised of urban residents, based upon which we can assume that upper muscle function is not so important for everyday life, for example, as it might be in rural areas, and this does not help to distinguish levels of HRQoL that well. Considering our results for HRQoL, we observed a significantly weaker independent association between GS and HRQoL domains (30-CST explained almost $30 \%$, while GS only around $10 \%$, of variance of PCS and MCS); this difference also remained in the comparison of males and females. This might also be one explanation as to why GS has not remained significant in adjusted models for men.

The findings of our study demonstrated that in the Estonian population, men's self-assessment of their health is substantially better. The reasons for such a difference between genders might be the age gap and the considerably low number of men with self-reported chronic diseases compared with women. An increase in the number of chronic diseases has been reported to have a negative impact on HRQoL [15]. In a study conducted in Sweden [16] to examine gender differences in the interpretation of self-rated health, chronic disease explained twice as much of the variance of regression analyses in self-rated health for women versus men. Furthermore, they found that overall general physical activity was more important for men, while for women, having a chronic disease was associated with lower self-reported health [15]. Still, unlike the previous study, our results demonstrated that the gender difference in values of HRQoL cannot be explained by systemic inflammation, as there was no significant difference in hsCRP for both genders [17]. 
The present study has several strengths. These strengths include simple physical function tests with good reproducibility $[9,10]$ and a high participation rate. From the initial sample, $95 \%$ of the subjects answered the invitation, based upon which the Estonian population representation (subjects per capita) was 1:3,979 [18]. As in our study, previously published data has shown that men usually perform muscle function tests somewhat better than women at each age $[19,20]$.

In addition to strengths, there are also some limitations that should be recognized. Firstly, a specific value on the SF-36 scale, which determines the low HRQoL and is applicable for different study populations, is not uniquely defined. Thus, it is questionable to divide SF-36 domains on the basis of lower quartile to define risk groups, and this is why the threshold values of 30-CST can be used only in the Estonian population. Secondly, self-reported data about chronic diseases does not allow for drawing precise conclusions on the impact of chronic disease on HRQoL, because a patient's understanding of chronic disease might largely differ from a doctor's opinion. Still, the wide range of symptoms and conditions that patients considered as chronic disease allows us to state that even a minor or short-term problem with health has a great impact on HRQoL. Finally, the cross-sectional design does not allow us to make causal inferences regarding associations found between self-reported HRQoL and physical function tests, but our observations should help in building up future studies.

\section{Conclusions}

We have demonstrated that physical function measures are associated with outcomes in self-reported HRQoL. The findings provide information that lower body strength is more strongly associated with $\mathrm{HRQ}$ oL than upper body strength, as higher results of 30-CST are significantly correlated with an increase in both physical and mental HRQoL. Additionally, this study emphasizes the concept that self-reported HRQoL is associated with different factors at the same time. With muscle function, factors such as age, systemic inflammation and chronic diseases become significant in the current health assessment. Taken together, 30-CST is a potential screening indicator for HRQoL assessment in clinical practice.

Acknowledgements. We would like to thank MSc Liis Starkopf for her valuable help in statistical analyses during the study.

Ethical approval. Ethics Review Committee on Human Research, University of Tartu.

Source of funding: This study was funded by the European Regional Development Fund through the Estonian Research Council's health research promotion program TerVE (grant number 3.2.1002.11-0002) and by Institutional Research (grant IUT 2-8).

Conflict of interest: The authors declare no conflict of interests.

\section{References}

1. Kielbergerová L, Mayer O Jr, Vaněk J, et al. Quality of life predictors in chronic stable post-stroke patients and prognostic value of SF-36 score as a mortality surrogate. Trans/ Stroke Res 2015; 6(5): 375-383.

2. Kanecki K, Nitsch-Osuch A, Tyszko PZ. Health-Related Quality of Life or Quality of Medical Service? Current challenges for family doctor. Fam Med Prim Care Rev 2016; 18(3): 382-386.

3. Halaweh H, Willen C, Grimby-Ekman A, et al. Physical Activity and Health-Related Quality of Life among community dwelling elderly J Clin Med Res 2015; 7(11): 845-852.

4. Cannell JJ, Grant WB, Holick MF. Vitamin D and inflammation. Dermatoendocrinol 2014; 6(1): e983401.

5. Sousa AC, Zunzunegui MV, Li A, et al. Association between C-reactive protein and physical performance in older populations: results from the International Mobility in Aging Study (IMIAS). Age Ageing 2016; 45(2): 274-280.

6. Cooper R, Kuh D, Hardy R, Mortality Review Group. Objectively measured physical capability levels and mortality: systematic review and meta-analysis. BMJ 2010; 341: c4467.

7. Svantesson U, Jones J, Wolbert K, et al. Impact of physical activity on the Self-Perceived Quality of Life in non-frail older adults. J Clin Med Res 2015; 7(8): 585-593.

8. Unver B, Kalkan S, Yuksel E, et al. Reliability of the 50-foot walk test and 30-sec chair stand test in total knee arthroplasty. Acta Ortop Bras 2015; 23(4): 184-187.

9. Fess EE. Grip strength. $2^{\text {nd }}$ edition. Chicago: American Society of Hand Therapists; 1992.

10. Bohannon RW, Schaubert KL. Test-retest reliability of grip-strength measures obtained over a 12-week interval from community-dwelling elders. J Hand Ther 2005; 18(4): 426-428.

11. Lai T, Kallikorm R, Salupere R, et al. Patsientide hinnangud oma tervisele krooniliste haiguste korral. Eesti Arst 2001; 80: 450-455.

12. Kull M, Kallikorm R, Tamm A, et al. Seasonal variance of $25-(\mathrm{OH})$ vitamin $\mathrm{D}$ in the general population of Estonia, a Northern European country. BMC Public Health 2009; 9: 22, doi: 10.1186/1471-2458-9-22.

13. Cooper R, Stafford M, Hardy R, et al. Physical capability and subsequent positive mental wellbeing in older people: findings from five HALCyon cohorts. Age 2014; 36(1): 445-456.

14. Hall SA, Chiu GR, Williams RE, et al. Physical function and health-related quality-of-life in a population-based sample. Aging Male 2011; 14(2): 119-126.

15. Põlluste K, Aart A, Kallikorm R, et al. Adverse lifestyle and health-related quality of life: gender differences in patients with and without chronic conditions. Scand J Public Health 2016; 44(2): 209-216.

16. Undén AL, Elofsson S. Do different factors explain self-rated health in men and women? Gend Med 2006; 3(4): $295-308$.

17. Garvin P, Nilsson E, Ernerudh J, et al. The joint subclinical elevation of CRP and IL-6 is associated with lower health-related quality of life in comparison with no elevation or elevation of only one of the biomarkers. Qual Life Res 2016; 25(1): 213-221.

18. Statistics Estonia (SE) [cited 18.10.2016]. Available at URL: http://www.stat.ee/ee/.

19. Mathiowetz V, Kashman N, Volland G, et al. Grip and pinch strength: normative data for adults. Arch Phys Med Rehabil 1985; 66(2): 69-72.

20. Bohannon RW, Bubela DJ, Magasi SR, et al. Sit-to-stand test: performance and determinants across the age-span. Isokinet Exerc Sci 2010; 18(4): 235-240.

Tables: 2

Figures: 2

References: 20 
Received: 23.12.2016

Revised: 08.01.2017

Accepted: 12.01.2017

Address for correspondence:

Ann Starkopf, MD

OÜ Perearst Anu Starkopf

Primary Health Care Centre

Nõlvaku 13-1

Tartu 50708

Estonia

Tel.: +37256691531

E-mail: starkopf.ann@gmail.com 